\title{
Blinded Role Code
}

National Cancer Institute

\section{Source}

National Cancer Institute. Blinded Role Code. NCI Thesaurus. Code C93519.

A coded value specifying the roles of individuals who are masked for blinded studies. 